\title{
Radial-breathing-like phonon modes of double-walled carbon nanotubes
}

\author{
Gang $\mathrm{Wu}$ * Jian Zhou, and Jinming Dong \\ Group of Computational Condensed Matter Physics, \\ National Laboratory of Solid State Microstructures and Department of Physics, \\ Nanjing University, Nanjing 210093, P. R. China
}

(Dated: November 16, 2018)

\begin{abstract}
The radial-breathing-like phonon modes (RBLMs) of the double-walled carbon nanotubes are studied in a simple analytical model, in which the interaction force constants (FCs) can be obtained analytically from the continuous model. The RBLMs frequencies are obtained by solving the dynamical matrix, and their relationship with the tube radii can be obtained analytically, offering a powerful experimental tool for determining precisely the radii of the multi-walled carbon nanotubes.
\end{abstract}

PACS numbers: $63.22 .+\mathrm{m}, 78.30 . \mathrm{Na}$

Right now, the double-walled carbon nanotubes (DWCNTs) become an important research subject after they are successfully synthesized by catalytic chemical vapor deposition ${ }^{1,2,3}$ and the thermal conversion of $\mathrm{C}_{60}$ encapsulated in single-walled carbon nanotubes (SWCNTs) $\underline{\underline{4}}$. Many researches $2,3,4,5,6,7,8,9,10,11,12,13$ have shown that there exists a kind of radial motion in the DWCNTs similar to the radial breathing modes (RBMs) in the SWCNTs, called as the radial breathing-like modes (RBLMs). But their relationship with the tube radii is still an open question, which is the most important for understanding the experimental RBLMs. In some publications $6,7,8,9,10,11,12$, the force constants are computed numerically, making the relationship between the RBLM frequencies and the tube radii unclear. Dobardžić et al. suggested a simple analytical model ${ }^{13}$, considering the tube walls as coupled oscillators, and the $\mathrm{FC}$ values of the coupled oscillators are determined by a fitting to the experimental $\mathrm{B}_{2 g}$ graphite phonon frequency of 127 $\mathrm{cm}^{-1}$. With this model, they have successfully calculated the RBLM frequencies of the DWCNT.

In this work, we have investigated further the RBLMs of the DWCNTs, using the simple analytical model ${ }^{13}$, and assuming the carbon atoms on the nanotube wall are distributed continuously, which has been used in a lot of systems $14,15,16,17,18,19$. Based on the continuum model in Ref. 19, we have derived a set of formulae to describe the interaction between an infinite length tube and an atom in any place. With these formulae, the force constants between the inner and outer tubes can be obtained analytically, and furthermore, an accurate relationship between the RBLM frequencies and the tube radii has been obtained. Our final results only involve a few basic parameter values relying on the nanotubes structure and the parameters of Lennard-Jones (LJ) potential.

Now, let us introduce the simple analytical model. For the RBLMs, the only degree of freedom is the motion along the radial direction of the tube, so we can simply consider the RBLMs as the motion of a harmonic oscillator. A segment of SWNT can be described as a smooth cylinder with its radius and height represented by $r$ and $h$. Then its Hamiltonian can be written as:

$$
H^{S}=\frac{1}{2} m \dot{r}^{2}+\frac{1}{2} m \omega_{R B M}^{2} r^{2} .
$$

Here the superscript $S$ means the SWNT. $m=$ $(2 \pi r h) n_{\sigma} m_{c}$ is the mass of the cylinder, $n_{\sigma}$ is the mean surface density of carbon atoms on the tube wall, and $m_{c}$ is the mass of carbon atom. $\dot{r}$ is the time derivative of the radius, and $\omega_{R B M}$ is the vibration frequency of the RBM mode. It is well known that the RBM frequency of the SWNT satisfies a simple relationship with the nanotube radius,

$$
\omega_{R B M}=D /\left(2 r_{0}\right),
$$

where $D$ keeps a constant for any kind of SWNTs and $r_{0}$ is the equilibrium radius of the SWNT. Thus we have

$$
H^{S}=\frac{1}{2} m \dot{r}^{2}+\frac{1}{8} m D^{2}\left(\frac{r}{r_{0}}\right)^{2} .
$$

For the DWCNTs, the Hamiltonian can be further written as 13 :

$$
H^{D}=H_{\text {out }}^{S}+H_{\text {in }}^{S}+V_{i o},
$$

where $H_{\text {out }}^{S}$ and $H_{\text {in }}^{S}$ are the Hamiltonians of the outer and inner SWNTs, respectively, with their radii as $r_{1}$ and $r_{2}$. And $V_{i o}$ is the interaction potential between the inner and outer tubes.

In this work, we assume the interaction between innerand outer-tube atoms can be described by the LennardJones(LJ) potential,

$$
u(x)=4 \varepsilon\left[-\left(\frac{\sigma}{x}\right)^{6}+\left(\frac{\sigma}{x}\right)^{12}\right]
$$

with parameters, $\varepsilon=2.964 \mathrm{meV}$ and $\sigma=3.407 \AA^{12}$. Based on our previous work ${ }^{19}$, when the tube height is large enough, the interaction potential can be writtend as 


$$
\begin{aligned}
V_{\text {io }}=\quad & N_{\text {in }} * 3 \pi r_{1} \varepsilon n_{\sigma} * V_{K E R N E L} \\
& +N_{\text {out }} * 3 \pi r_{2} \varepsilon n_{\sigma} * V_{K E R N E L}
\end{aligned}
$$

where

$$
\begin{aligned}
& V_{K E R N E L}=\left[-\frac{\sigma^{6}}{\left(4 r_{1} r_{2}\right)^{\frac{5}{2}}} I_{5}+\frac{21}{32} \frac{\sigma^{12}}{\left(4 r_{1} r_{2}\right)^{\frac{11}{2}}} I_{11}\right] \\
& I_{2 k+1}=\int_{0}^{\frac{\pi}{2}} \frac{d t}{\left(a^{2}+\sin ^{2} t\right)^{\frac{2 k+1}{2}}}, \quad a^{2}=\frac{\left(r_{1}-r_{2}\right)^{2}}{4 r_{1} r_{2}} .
\end{aligned}
$$

$N_{\text {in }}=2 \pi r_{2} n_{\sigma} h$ and $N_{\text {out }}=2 \pi r_{1} n_{\sigma} h$ are the number of atoms of the inner and outer tube segment, respectively. $k$ is an integer.

For the DWCNTs, when inner tube radius $r_{2}$ is larger than $\left(r_{1}-r_{2}\right) \approx 3.4 \AA$, the $a^{2} \leq \frac{1}{8}$, so we can use our series expression ${ }^{19}$ for the Eq. (8), and get

$$
I_{2 k+1} \approx\left\{\frac{1}{(2 k-1) ! !}\left(\frac{2}{a^{2}}\right)^{k} \sum_{m=0}^{k-1} \frac{[(2 m) !]^{2}}{[m !]^{3}} \frac{(k-m-1) !}{2}\left(\frac{a}{4}\right)^{2 m}+\frac{(2 k-1) ! !}{(2 k) ! !}\right\} .
$$

Now we can obtain the dynamical matrix analytically,

$$
F=\left[\begin{array}{ll}
\frac{1}{m_{\text {out }}} \frac{\partial^{2} H^{D}}{\partial r_{1}^{2}} & \frac{1}{\sqrt{m_{\text {out }} m_{\text {in }}}} \frac{\partial^{2} H^{D}}{\partial r_{1} \partial r_{2}} \\
\frac{1}{\sqrt{m_{\text {in }} m_{\text {out }}}} \frac{\partial^{2} H_{2} \partial}{\partial r_{1}} & \frac{1}{m_{\text {in }}} \frac{\partial^{2} H^{D}}{\partial r_{2}^{2}}
\end{array}\right]=\left[\begin{array}{ll}
\left(\omega_{R B M}^{\text {out }}\right)^{2} & 0 \\
0 & \left(\omega_{R B M}^{\text {in }}\right)^{2}
\end{array}\right]+\left[\begin{array}{ll}
K_{11} & K_{12} \\
K_{21} & K_{22}
\end{array}\right]
$$

Here,

$$
\begin{gathered}
K_{11}=\frac{1}{m_{\text {out }}} \frac{\partial^{2} V_{\text {io }}}{\partial r_{1}^{2}} \approx C_{0}\left[\frac{88}{3} s_{0}^{12}-\frac{40}{3} s_{0}^{6}-\left(\frac{44}{3} s_{0}^{11}-\frac{20}{3} s_{0}^{5}\right) s_{1}-\left(\frac{33}{10} s_{0}^{10}-\frac{5}{4} s_{0}^{4}\right) s_{1}^{2}+\ldots\right] \\
K_{21}=K_{12}=\frac{1}{\sqrt{m_{\text {out }} m_{\text {in }}}} \frac{\partial^{2} V_{\text {io }}}{\partial r_{1} \partial r_{2}} \approx C_{0}\left[-\frac{88}{3} s_{0}^{12}+\frac{40}{3} s_{0}^{6}+\left(-\frac{11}{30} s_{0}^{10}+\frac{5}{12} s_{0}^{4}\right) s_{1}^{2}+\ldots\right], \\
K_{22}=\frac{1}{m_{\text {in }}} \frac{\partial^{2} V_{\text {io }}}{\partial r_{2}^{2}} \approx C_{0}\left[\frac{88}{3} s_{0}^{12}-\frac{40}{3} s_{0}^{6}+\left(\frac{44}{3} s_{0}^{11}-\frac{20}{3} s_{0}^{5}\right) s_{2}-\left(\frac{33}{10} s_{0}^{10}-\frac{5}{4} s_{0}^{4}\right) s_{2}^{2}+\ldots\right]
\end{gathered}
$$

where $s_{0}=\frac{\sigma}{d_{0}}, s_{1}=\frac{\sigma}{r_{1}}$, and $s_{2}=\frac{\sigma}{r_{2}} . d_{0}=r_{1}-r_{2}$ is the distance between the inner and outer tubes. Above formulae are suitable for any tube-tube distance.

Assuming $d_{0}=3.4 \AA \approx \sigma$, which is equal to the interlayer spacing of graphite, we can further get

$$
\begin{aligned}
K_{11} & =\frac{1}{m_{\text {out }}} \frac{\partial^{2} V_{\text {io }}}{\partial r_{1}^{2}} \\
& \approx C_{0}\left[16-8 s_{1}-\frac{41}{20} s_{1}^{2}-\frac{41}{40} s_{1}^{3}+\ldots\right]
\end{aligned}
$$

$$
\begin{aligned}
K_{21} & =K_{12}=\frac{1}{\sqrt{m_{\text {out }} m_{\text {in }}}} \frac{\partial^{2} V_{\text {io }}}{\partial r_{1} \partial r_{2}} \\
& \approx C_{0}\left[-16+\frac{1}{20} s_{1}^{2}+\frac{1}{20} s_{1}^{3}+\ldots\right]
\end{aligned}
$$

$$
\begin{aligned}
K_{22} & =\frac{1}{m_{i n}} \frac{\partial^{2} V_{i o}}{\partial r_{2}^{2}} \\
& \approx C_{0}\left[16+8 s_{2}-\frac{41}{20} s_{2}^{2}+\frac{41}{40} s_{2}^{3}+\ldots\right] .
\end{aligned}
$$

Here $C_{0}=\frac{3 \pi n_{\sigma} \varepsilon}{m_{c}}$. Choosing $n_{\sigma}=\frac{4}{3 \sqrt{3}} \frac{1}{a_{C-C}^{2}}$, with $a_{C-C}$ the bond length of the SWNT, being about 1.42 $\AA, \varepsilon=2.964 \mathrm{meV}$, and $m_{c}=12.011 \mathrm{amu}$, we can obtain $C_{0} \approx 241 \mathrm{~cm}^{-2}$. In this work, the prefactor $D$ in Eq. 2 
is always chosen as $225 \mathrm{~cm}^{-1} \mathrm{~nm}$. By diagonalizing the dynamical matrix of Eq. 10, the RBLM frequencies can be obtained.

For example, the RBLM frequencies of $(5,5) @(10,10)$ DWCNT are calculated using above $C_{0}$ and $D$. Then one can easily obtain $\omega_{L F}=173.9 \mathrm{~cm}^{-1}$ and $\omega_{H F}=340.8$ $\mathrm{cm}^{-1}$. And the corresponding RBM frequencies of $(5,5)$ and $(10,10)$ SWNTs obtained by Eq. 2 are $\omega_{(5,5)}=331.9$ $\mathrm{cm}^{-1}$ and $\omega_{(10,10)}=165.9 \mathrm{~cm}^{-1}$. Thus the frequency shifts of the RBLMs in the DWCNT are $\Delta \omega_{L F}^{L J}=\omega_{L F}-$ $\omega_{(10,10)}=8.0 \mathrm{~cm}^{-1}$ and $\Delta \omega_{H F}^{L J}=\omega_{H F}-\omega_{(5,5)}=8.9$ $\mathrm{cm}^{-1}$, respectively.

To make a comparison, a first-principle calculation has been performed on the $(5,5) @(10,10)$ DWNT to check the validity of our method. A supercell geometry was adopted so that the DWCNT is aligned in a hexagonal array with nearby DWCNT center distance of $25 \AA$, which is found to be larger enough to prevent the tube-tube interactions. The K-points sampling in the reciprocal space is a uniform grid $1 \times 1 \times 24$ along the nanotube axis $(Z$ direction) in our calculations. After structure relaxation on both of the lattice constant along the tube axis and the atomic positions, the optimal structure is obtained when the residue forces acting on all the atoms were less than $0.01 \mathrm{eV} / \AA$. The final dynamical matrix is constructed using cumulant force-constant (CFC) method 20,21 . Because of the advantage of the CFC method in dealing with the low frequency part of the phonon dispersion, only a small supercell $(1 \times 1 \times 2)$ is sufficient to calculate accurately the phonon modes on the $\Gamma$ point and the $X$ point. Our $a b$ initio calculations were performed using highly accurate projected augmented wave (PAW) $\operatorname{method}^{22}$, implemented in the Vienna ab initio simulation package (VASP) package $e^{23}$. They are based on the density-functional theory in the local-density approximation (LDA).

The obtained phonon dispersion curves and the density of states for the $(5,5) @(10,10)$ DWCNT are shown in Fig. 1, in which the low-frequency dispersions are very well reproduced. Its RBLM frequencies can be determined by analysis of the normal modes, which are $\omega_{L F}^{a b}=182.2 \mathrm{~cm}^{-1}$ and $\omega_{H F}^{a b}=354.6 \mathrm{~cm}^{-1}$. The RBM frequencies of $(5,5)$ and $(10,10)$ SWNTs are also calculated by the same $a b$ initio method under the same calculation conditions as the DWCNT, which are $\omega_{(5,5)}^{a b}=343.8 \mathrm{~cm}^{-1}$ and $\omega_{(10,10)}^{a b}=173.6 \mathrm{~cm}^{-1}$. Then, the corresponding frequency shifts of them in the DWCNT are $\Delta \omega_{L F}^{a b}=\omega_{L F}^{a b}-\omega_{(10,10)}^{a b}=8.6 \mathrm{~cm}^{-1}$ and $\Delta \omega_{H F}^{a b}=\omega_{H F}^{a b}-\omega_{(5,5)}^{a b}=10.8 \mathrm{~cm}^{-1}$, respectively. These results are consistent with our above obtained results. The residual difference can be further minimized by adopting the first-principle tube radii of $R_{(5,5)}=3.411$ $\AA$ and $R_{(10,10)}=6.766 \AA$, and the first-principle RBM frequencies of the SWNTs in the dynamical matrix of Eq. 10. By this way, the new frequency shifts obtained by our analytical method will be $\Delta \omega_{L F}^{L J}=9.0 \mathrm{~cm}^{-1}$ and $\Delta \omega_{H F}^{L J}=10.3 \mathrm{~cm}^{-1}$, agreeing very well with above first-
FIG. 1: (Color online) Calculated phonon dispersion and the density of states for the $(5,5) @(10,10)$ are shown in the left and right panels, respectively.

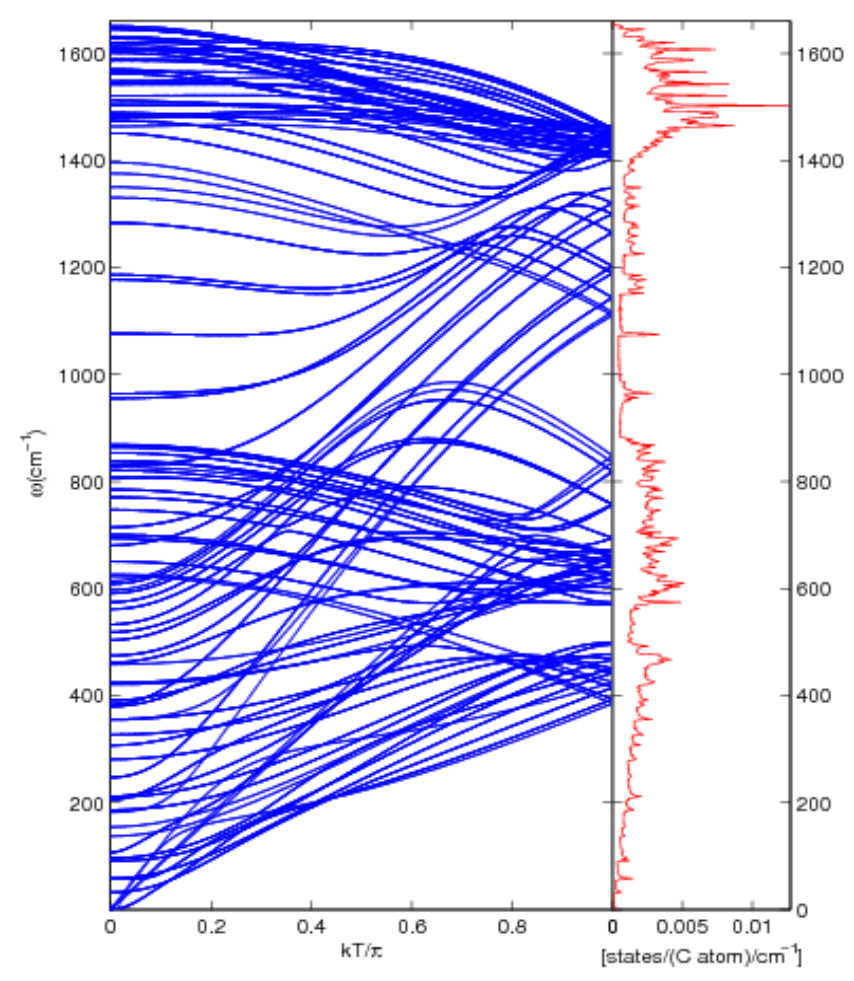

principle values.

Our approach can be further applied to the multiwalled carbon nanotubes (MWCNTs). Here we take a 3-layer MWCNT as an example. Again, we can write the system Hamiltonian as

$$
H^{T}=\sum_{i=1}^{3} H_{i}^{S}+\sum_{i \neq j} V_{i j}
$$

where $H_{i}^{S}$ is the Hamiltonian of the $i$ th layer, and $V_{i j}$ is the interaction potential between the $i$ th and the $j$ th layer nanotubes. And the radius of the $i$ th layer is represented by $r_{i}$ with $r_{1}>r_{2}>r_{3}$. So, its dynamical matrix can be written as: 


$$
\begin{aligned}
F= & {\left[\begin{array}{lll}
\frac{1}{m_{1}} \frac{\partial^{2} H^{T}}{\partial r_{1}^{2}} & \frac{1}{\sqrt{m_{1} m_{2}}} \frac{\partial^{2} H^{T}}{\partial r_{1} \partial r_{2}} & \frac{1}{\sqrt{m_{1} m_{3}}} \frac{\partial^{2} H^{T}}{\partial r_{1} \partial r_{3}} \\
\frac{1}{\sqrt{m_{2} m_{1}}} \frac{\partial^{2} H^{T}}{\partial r_{2} \partial r_{1}} & \frac{1}{m_{2}} \frac{\partial^{2} H^{T}}{\partial r_{2}^{2}} & \frac{1}{\sqrt{m_{2} m_{3}}} \frac{\partial^{2} H^{T}}{\partial r_{2} \partial r_{3}} \\
\frac{1}{\sqrt{m_{3} m_{1}}} \frac{\partial^{2} H^{T}}{\partial r_{3} \partial r_{1}} & \frac{1}{\sqrt{m_{3} m_{2}}} \frac{\partial^{2} H^{T}}{\partial r_{3} \partial r_{2}} & \frac{1}{m_{3}} \frac{\partial^{2} H^{T}}{\partial r_{3}^{2}}
\end{array}\right] } \\
= & {\left[\begin{array}{lll}
\left(\omega_{R B M}^{1}\right)^{2} & 0 & 0 \\
0 & \left(\omega_{R B M}^{2}\right)^{2} & 0 \\
0 & 0 & \left(\omega_{R B M}^{3}\right)^{2}
\end{array}\right] } \\
& +\left[\begin{array}{lll}
K_{11}^{12}+K_{11}^{13} & K_{12}^{12} & K_{13}^{13} \\
K_{21}^{12} & K_{22}^{12}+K_{22}^{23} & K_{23}^{23} \\
K_{31}^{13} & K_{32}^{23} & K_{33}^{13}+K_{33}^{23}
\end{array}\right],(14)
\end{aligned}
$$

where $K_{\alpha \beta}^{i j}$ means the $(\alpha, \beta)$ element of the $K$ matrix, which comes from the interaction between the $i$ th and the $j$ th layer tubes. If condition $a_{13}^{2}=\frac{\left(r_{1}-r_{3}\right)^{2}}{4 r_{1} r_{3}} \ll 1$ can be satisfied, the Eq. (11) can also be used to get the force constants between the 1st and the 3rd layer tubes. So using Eq. (11), the force constants between any two different layer tubes can be obtained easily. Here, for the 3 layers MWNT, we use $d_{0}=6.8 \AA$ as the distance between the 1st and the 3rd layer tubes, then Eq. (14) is fully determined.

To examine the precision of our result, we compare them with other numerical ones. The RBLM frequencies as a function of the outer tube radius for $(n, n) @(n+5, n+5) @(n+10, n+10)$ MWCNTs are plotted in Fig. 2 (For comparison, please see figure 5 of Ref. 12). From Fig. 2, it is found that our results match Ref. 12 very well, and the residual difference may come from the choice of some parameters, e.g., $D, d_{0}$ and $n_{\sigma}$. In fact, if we only slightly adjust both of $D$ and $C_{0}$, our RBLM frequencies can match all the other publications $6.7,8,9,10,11,12$ perfectly.

FIG. 2: (Color online) Dependence of the RBLM freqeuncies on the outermost tube radius for 3 layer MWCNTs, i.e., $(n, n) @(n+5, n+5) @(n+10, n+10)$. The highest (HF), the middle (MF) and the lowest (LF) RBLM are plotted.

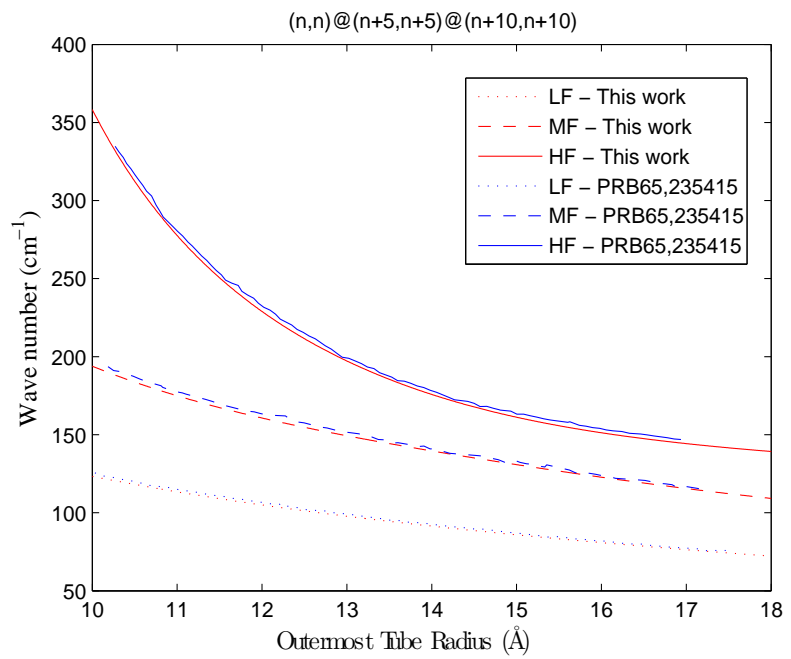

Furthermore, we show the dependence of the RBLM frequeuncies on the number of layers in Fig. 3, in which the radius of the innermost tube is fixed at $30 \AA$. This result matches the Fig. 6 of Ref. 12 perfectly. Based upon Fig. 3, a new method to determine the number of layers for the MWCNTs can be proposed, i.e., if the position of the lowest RBLM for a MWNT is obtained, one can easily find out how many layers are present in the MWCNT by comparing the experimental data with Fig. 3.

FIG. 3: (Color online) Dependence of the RBLM freqeuncies on the layer number. Here, the radius of the innermost tube is fixed at $30 \AA$.

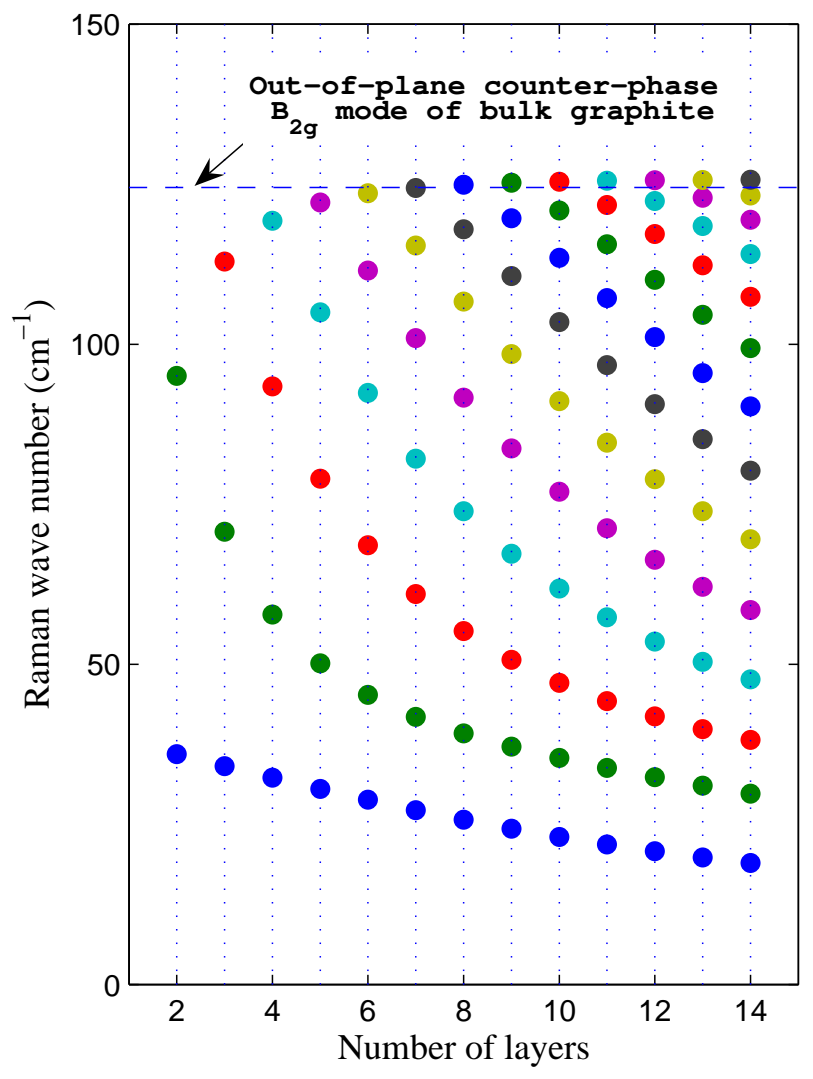

Now we would like to illustrate the application of our approach. In Ref. 5, the Raman investigation of the DWCNTs prepared from thermal conversion of $\mathrm{C}_{60}$ encapsulated in the SWCNTs has been reported, in which the Eq. (2), suitable only to the SWCNTs, was used to obtain the tube radii. Now we used our approach to determine the inner tube radius and the tube-tube distance. Here we choose a pair of experimental data from Ref. [5, $\omega_{L F}=150 \mathrm{~cm}^{-1}$, and $\omega_{H F}=267 \mathrm{~cm}^{-1}$. By Eq. (11), the high and low RBLM frequencies for different $r_{2}$ can be obtained at the certain $d_{0}$. Then comparing them with the experimental frequencies, we can get two different inner tube radii. In a real system, these two inner 
tube radii value must be the same, and so we have to adjust the distance $d_{0}$ between the inner and outer tubes. Finally, a most reliable inner tube radius can be found. In Fig. 4, we finally set $d_{0}$ to be $3.558 \AA$, making the cross points of low and high frequencies with the experimental frequencies lie on one vertical line, from which a reliable inner tube radius of about $4.453 \AA$ is obtained, i.e., $R_{\text {in }}=4.453 \AA$, and so $R_{\text {out }}=8.011 \AA$.

FIG. 4: (Color online) The Raman frequency vs the inner tube radius, which is obtained after adjusting the distance $d_{0}$ between the inner and outer walls to about $3.558 \AA$.

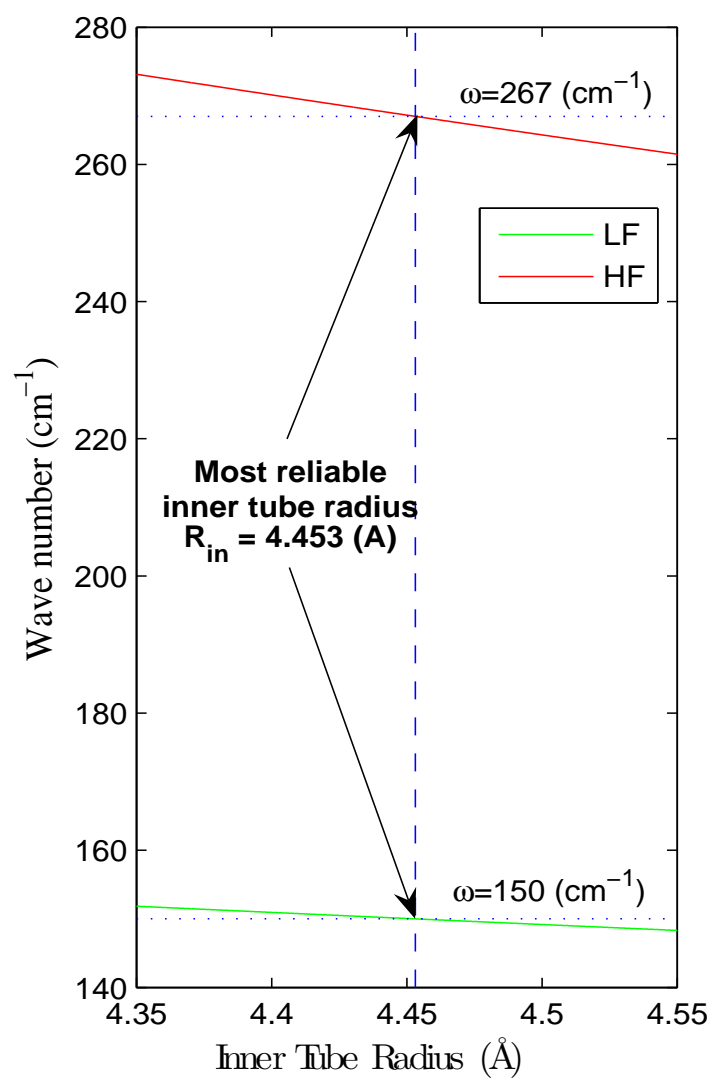

In summary, we presented an analytically solvable approach for the radial breathing-like phonon modes of the MWNTs, where the tube walls are treated as coupled oscillators, and the carbon atoms on nanotubes are distributed continuously. Then the force constants between inner and outer tubes, and the relationship of the RBLM frequencies with the tube radii can be obtained analytically. It is found that our obtained RBLM frequencies are well consistent with those in other publications and experiments. Finally, our result can be helpful to determine the radii of the DWCNTs and MWCNTs.

\section{Acknowledgments}

The authors acknowledge support from the Natural Science Foundation of China under Grants No. 10474035 and No. A040108. The authors also thanks support to this work from a Grant for State Key Program of China through No. 2004CB619004. Our first-principle calculations have been done on the Sun Fire V20z computers.
* Electronic address: E-mail: wugaxp@gmail.com

$\dagger$ Corresponding author E-mail: jdong@nju.edu.cn

1 T. Sugai, H. Omote, S. Bandow, N. Tanaka, and H. Shinohara, J. Chem. Phys. 112, 6000 (2000).

2 E. Flahaut, A. Peigney, Ch. Laurent, A. Rousset, J. Mater. Chem. 10, 249 (2000).

${ }^{3}$ H. Zhu, C. Xu, B. Wei, and D. Wu, Carbon 40, 2021 (2002).

4 S. Bandow, M. Takizawa, H. Hirahara, M.Yudasaka, and S. Iijima, Chem. Phys. Lett. 337, 48 (2001).

5 S. Bandow, G. Chen, G. U. Sumanasekera, R. Gupta, M. Yudasaka, S. Iijima, and P. C. Eklund, Phys. Rev. B 66, 075416 (2002).
${ }^{6}$ E. Dobardžić, I. Milošević, T. Vuković, B. Nikolić, and M. Damnjanović, Eur. Phys. J. B 34, 409 (2003).

7 M. Damnjanović, I. Milošević, E. Dobardžić, T. Vuković, and B. Nikolić, Phys. Rev. B 69, 153401 (2004).

8 M. Damnjanović, E. Dobardžić, I. Milošević, T. Vuković, and B. Nikolić, New J. Phys. 5, 148 (2003).

9 M. Xia, S. Zhang, E. Zhang, S. Zhao, and X. Zuo, Phys. Rev. B 69, 233407 (2004).

${ }^{10}$ R. Pfeiffer, Ch. Kramberger, F. Simon, H. Kuzmany, V.N. Popov, and H. Kataura, Eur. Phys. J. B 42, 345 (2004).

11 A. Rahmani, J.-L. Sauvajol, J. Cambedouzou, and C. Benoit, Phys. Rev. B 71, 125402 (2005).

12 V. N. Popov and Luc Henrard, Phys. Rev. B 65, 235415 
(2002).

13 E. Dobardžić, J. Maultzsch, I. Milošević, C. Thomsen, M. Damnjanović, Phys. Status Solidi B 237 (2), R7 (2003).

14 L.A. Grrifalco, J. Phys. Chem 96, 858 (1992); L.A. Girifalco, Phys. Rev. B 52, 9910 (1995).

15 C. Rey, J. Garcia-Rodeja, L.J. Gallego, J.A. Alonso, Phys. Rev. B 55, 7190 (1997).

16 J. Song and R.L. Cappelletti, Phys. Rev. B 50, 14678 (1994).

17 L. Henrard, E. Hernandez, P. Bernier, and A. Rubio, Phys. Rev. B 60, R8521 (1999).

18 L.A. Girifalco, Miroslav Hodak, and Roland S. Lee, Phys.
Rev. B 62, 13104(2000).

19 G. Wu and J. Dong, Phys. Rev. B 71, 115410 (2005).

${ }^{20}$ K. Parlinski, Z-.Q. Li, and Y. Kawazoe, Phys. Rev. Lett. 78, 4063 (1997)

21 L.H. Ye, B.G. Liu, D.S. Wang, and R. Han, Phys. Rev. B 69, 235409 (2004).

22 P.E. Blöchl, Phys. Rev. B 50, 17953 (1994); G. Kresse and D. Joubert, ibid. 59, 1758 (1999).

23 G. Kresse and J. Hafner, Phys. Rev. B 48, 13115 (1993); G. Kresse and J. Furthmüller, Comput. Mater. Sci. 6, 15 (1996); Phys. Rev. B 54, 11169 (1996). 